\title{
Micromanaging autophagy with microRNAs to drive cancer metastasis
}

\author{
Gracie Wee Ling Eng ${ }^{1,2}$, Venetia Jing Tong Kok ${ }^{1}$, Jit Kong Cheong ${ }^{1,2}$ \\ 'Department of Biochemistry, Yong Loo Lin School of Medicine, National University of Singapore, Singapore 117597, Singapore. \\ ${ }^{2}$ Medical Sciences Cluster, Yong Loo Lin School of Medicine, National University of Singapore, Singapore 117597, Singapore.
}

Correspondence to: Prof. Jit Kong Cheong, Department of Biochemistry, Yong Loo Lin School of Medicine, National University of Singapore, Singapore. 8 Medical Drive, MD7 \#03-09, Singapore 117597, Singapore. E-mail: bchcjk@nus.edu.sg

How to cite this article: Eng GWL, Kok VJT, Cheong JK. Micromanaging autophagy with microRNAs to drive cancer metastasis. J Cancer Metastasis Treat2019;5:69. http://dx.doi.org/10.20517/2394-4722.2019.021

Received: 31 Jul 2019 First Decision: 6 Sep 2019 Revised: 17 Sep 2019 Accepted: 24 Sep 2019 Published: 30 Sep 2019

Science Editor: Chun Hei Antonio Cheung Copy Editor: Jia-Jia Meng Production Editor: Jing Yu

\begin{abstract}
While we made great strides in the early detection of a handful of cancers, many other cancers are still detected at fairly later stages, thus hindering the deployment of effective surgical or therapeutic intervention to change their dismal clinical outcomes. The arduous journey of cancer cells from the primary tumor to colonize distant secondary organs or tissues begins with their ability to activate or deactivate various cellular processes at will, including the autophagy machinery. In this review, we discuss how circulatory cancer cells from primary tumors could selectively mobilize different subsets of microRNAs (miRNAs) to enable autophagic recycling of nutrients during their search for secondary sites to colonize and to disable such cell survival programs once they have successfully established at distant organs or tissues. We also discuss how this new miRNA-autophagy-metastasis axis can be targeted by the emerging RNA Medicine toolkit.
\end{abstract}

Keywords: Autophagy, miRNAs, cancer, metastasis

\section{CANCER METASTASIS: KNOWING TOO LITTLE, TOO LATE}

Metastasis remains one of the key reasons for the poor prognosis of cancer patients. The one-year survival of patients with stage 1 localized lung cancers is $87.3 \%$, but plummets to merely $18.7 \%$ for stage 4 metastatic lung cancers ${ }^{[1]}$. Similar trend is observed for colorectal cancer, where one-year survival is $97.7 \%$ if detected at stage 1 and rapidly decline to $43.9 \%$ if detected at stage 4 . These depressing statistics underscores our

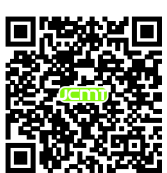


current knowledge gap in understanding the metastatic process, which impacts our ability to offer curative treatment options to cancer patients.

In the early stages of cancer, tumors are typically benign and remain confined within the normal boundaries of a tissue. As tumors grow and become malignant, however, they acquire the capacity to break through these boundaries and invade adjoining tissues. Notably, dissemination of tumor cells can occur relatively early in cancer progression ${ }^{[2]}$. During the process of metastasis, only a rare population of invasive cancer cells escape the primary tumor, survive the treacherous transit through the circulatory system, and subsequently establish secondary tumors in distant organs/tissues ${ }^{[3]}$. These invasive cancer cells, which exhibit extensive cytoskeletal remodeling, secrete proteases like extracellular-matrix-degrading metalloproteinases and cathepsins to drive their invasion and migration through the stroma, a network of supportive, connective tissue cells at the basement membrane $e^{[4,5]}$. Furthermore, the stroma can actively promote the initiation of metastasis by releasing transforming growth factor- $\beta$ and other cell-signalling proteins to trigger cancer cells to undergo epithelial-to-mesenchymal transition (EMT) - a reversible phenotypic change in which cells lose intercellular adhesion and epithelial polarization and gain motility and invasiveness ${ }^{[6]}$. Transition into a mesenchymal cancer cell state allows for efficient intravasation of the lymphovascular system and acquisition of a stem-cell phenotype to promote survival during transit. These circulatory cancer cells can be arrested at distant organs/tissues, where they extravasate into the parenchyma of target organs to commence colonization. After extravasation, the invading cancer cells undergo mesenchymal-to-epithelial transition (MET) at the new settlement sites. The conduciveness of these niches for colonization can dictate the period of dormancy of these micrometastatic cancer cells. Long period of latency often exists between the development of a primary tumor and clinical manifestations of metastasis, indicating that metastatic colonization is a highly inefficient process in which most of these disseminated tumor cells (DTCs) die. To survive and establish macrometastases at distant organs/tissues, the minor subset of DTCs must overcome immune surveillance and other hosttissue defenses to achieve overt colonization at secondary sites ${ }^{[7]}$. Although breast-to-lung metastases have been shown to arise mainly from cells that have not undergone EMT in mouse models of breast cancer ${ }^{[8]}$, it could be interpreted as rapid conversion into the epithelial cell state of DTCs, via MET, as soon as they arrive at supportive niches in the lung. Notably, the breast cancer cells that have undergone EMT are found to be resistant to chemotherapy and this could be attributed to their acquisition of a stem cell-like phenotype ${ }^{[9]}$.

\section{AUTOPHAGY: A CRUCIAL CANCER CELL SURVIVAL MECHANISM DURING METASTASIS}

Autophagy is a cellular housekeeping mechanism which degrades and recycles bulks of cytoplasmic materials for the synthesis of essential cellular components ${ }^{[10]}$. Highly conserved from yeast to mammals, autophagy acts as an adaptive response to environmental stresses such as nutrient deprivation. The autophagy machinery has multiple distinct stages: initiation, nucleation, elongation, fusion and cargo recycling ${ }^{[10]}$. During stress, shifts in nutrient availability can be detected by proteins involved in energy homeostasis, such as the $5^{\prime}$ AMP-activated protein kinase (AMPK ${ }^{[1]]}$ and the target of rapamycin complex 1 (TORC1). The activation of AMPK and the corresponding inhibition of TORC1 will lead to the assembly of the Atg1/ULK complex, thereby kickstarting autophagy.

During autophagy, cytoplasmic cargoes are encapsulated by double-membrane vesicles known as autophagosomes and thereafter sent to the lysosomes to be degraded ${ }^{[12]}$. The intricate, multistep processes of autophagy are facilitated by numerous autophagy-related proteins (Atg). Detailed characterization of these proteins can be found in the review by Feng et al.$^{[13]}$. At the initiation stage, the Atg1/ULK complex is assembled. This complex consists of Atg1/ULK, the regulatory subunit Atg13 and the scaffold complex Atg7-Atg31-Atg29 [Figure 1]. Importantly, the assembly of the Atg1/ULK complex is critical for the 


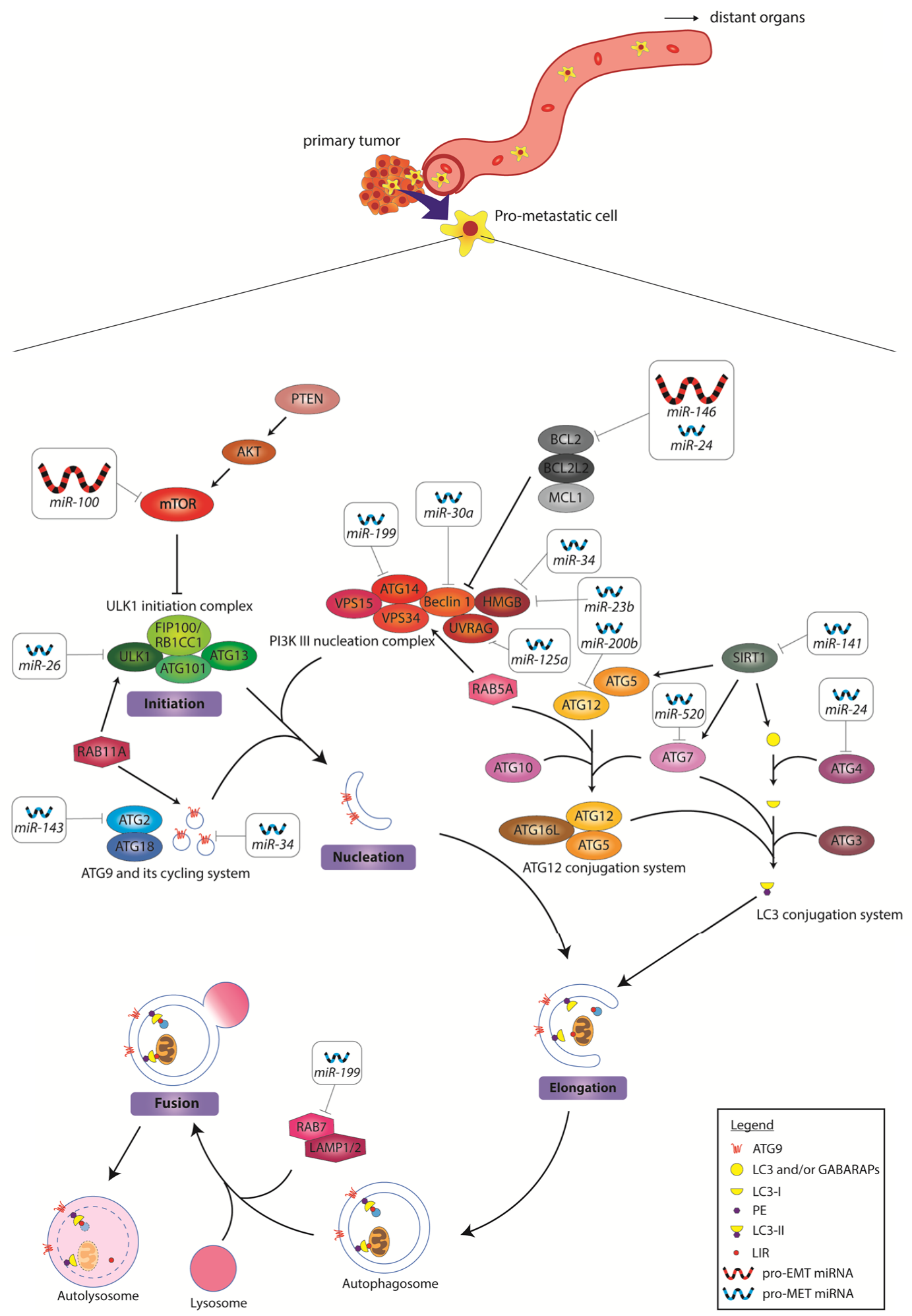

Figure 1. MiRNA regulation of autophagy during cancer invasion. The key components of macroautophagy is shown in this diagram. In the invading pro-metastatic cancer cell, autophagy is upregulated by gain of oncomiRs and loss of tumor-suppressive miRNAs. The oncomiRs target the inhibitors of autophagy to increase autophagic flux. The downregulated miRNAs on the other hand, are required for the activation of autophagy. Upregulated pro-EMT miRNAs are depicted as large red icons, while downregulated pro-MET miRNAs are depicted as small blue icons. The miRNA targets and mechanisms of actions are summarized in Table 1. EMT: epithelial-to-mesenchymal transition; oncomiRs: oncogenic miRNAs; MET: mesenchymal-to-epithelial transition 
recruitment of downstream proteins to the phagophore assembly site. Next, Atg9 and its cycling system (Atg2, Atg18) are activated to facilitate membrane delivery to the expanding phagophore. This is followed by the assembly of the PI3K complex consisting of Vps34, Vps15, Vps30/Atg6, Atg14, and Atg38 [Figure 1], a process responsible for recruiting more proteins that are required for further phagophore expansion. Subsequently, two ubiquitin-like conjugation systems are activated for phagophore expansion, namely the Atg12 and the Atg8 conjugation systems. A detailed mechanism in which these conjugation systems function has been reported by Yin et al. ${ }^{[10]}$. The human homologs of Atg8 are categorized into two families - LC3 and GABARAP. Importantly, LC3 is employed during the phagophore elongation stage, whereas GABARAP proteins are only activated at a later stage of maturation. Upon induction of phagophore elongation, LC3 is cleaved at the C-terminal by ATG4B protease to form cytosolic LC3-I. This is followed by its subsequent conjugation and recruitment to the membrane to form LC3-II ${ }^{[13]}$. The level of LC3-II is often known to correlate with the number of autophagosomes due to its association with autophagosome maturation $^{[14]}$.

Fundamentally, LC3-II acts as an adaptor protein that selectively recruits cargoes to the autophagosome to be degraded ${ }^{[14]}$. Following cargo recruitment and closure of the autophagosome, the autophagosome directly fuses with a lysosome to form an autolysosome ${ }^{[12]}$. The cytosolic cargoes within the autolysosome are then broken down by lysosomal hydrolases, liberating building blocks for future synthesis of other macromolecules.

The importance of autophagy as a cellular homeostatic mechanism is underscored by the prevalence of autophagic defects in a myriad of diseases. Besides being a protein and organelle quality control mechanism that prevents the accumulation of aggregated and dysfunctional proteins, basal autophagy is also a highly regulated catabolic process that supports cellular metabolic and biosynthetic programs in response to nutrient deprivation and other forms of stress. In cancer pathogenesis, however, autophagy is hijacked by rapidly proliferating cancer cells as they adapt to the perturbations of the cancer microenvironment ${ }^{[1]]}$.

Autophagy is finely regulated in response to the specific energy demands or stress cues emitted throughout the different stages of metastasis - local invasion, intravasation, dissemination, extravasation and colonization at distant sites. Several studies suggested that autophagy is associated with EMT in cancer and activation of autophagy promotes metastasis ${ }^{[16]}$. This is corroborated by the high levels of autophagy detected in EMT-activated cancer cells, hence strongly supporting its role in promoting early metastasis ${ }^{[17]}$. Additionally, cell detachment from the extracellular matrix typically has been shown to induce anoikis, a self-defense strategy that eliminates misplaced cells through apoptosis ${ }^{[18,19]}$. It then becomes crucial for cancer cells to evade anoikis so as to survive while traversing to distant organs/tissues. Importantly, several studies have postulated the pro-metastatic role of autophagy in overcoming anoikis ${ }^{[20-22]}$. Although the underlying mechanisms remain largely unknown, anoikis resistance conferred by autophagy has been shown to enhance the survival of cancer cells when they disseminate into the lymphovascular system, leading to their successful metastasis to distant organs.

Given that autophagy is a key cellular process that confers stress tolerance under adverse conditions, its activation in cancer cells that intravasate into the blood or lymphatic vessels enables them to withstand extreme metabolic stress induced by the changing environment ${ }^{[23]}$. To compensate for the sudden change in energy demands of the circulating DTCs, autophagy is activated to retain pools of functional mitochondria to sustain viability of the DTCs. In addition, autophagy-mediated degradation of proteins provides a sustainable source of nutrients for the DTCs to fuel ATP production and biosynthesis of other macromolecules that are essential for growth and survival ${ }^{[23,24]}$.

Successful metastatic colonization is not only limited to the ability of DTCs to overcome stress that they are exposed to during circulation. Following extravasation from the blood vessel, the DTCs have been shown 
to switch from EMT to MET with concurrent inactivation of autophagy in supportive niches ${ }^{[25]}$. However, if the new environment is not permissive in situations such as cellular stress or a lack of available growth factors, the DTCs could exist in a dormant state, possibly by retaining elevated autophagic functions and delaying MET. Notably, autophagy inhibition in the neoadjuvant setting has also been shown to reduce pro-metastasis stromal cells at the premetastatic niche ${ }^{[26]}$. Future work could be performed to determine whether inactivation of autophagy is necessary to drive MET of invading tumor cells at distant organs/ tissues, thus providing new impetus for the development of anti-metastasis autophagy inhibitors.

\section{MIRNAS: NONCODING REGULATORS OF AUTOPHAGY AND CANCER METASTASIS}

MicroRNAs (miRNAs) are endogenously expressed small non-coding RNAs of 19-25 nucleotides in length, which negatively regulate gene expression post-transcriptionally. They regulate multiple cellular processes including proliferation, differentiation and apoptosis. They have been implicated in a growing list of human diseases, such as diabetes and cancer ${ }^{[27]}$.

In the canonical miRNA biogenesis pathway, the primary transcript (pri-miRNA) in the nucleus forms a characteristic stem loop structure, which is recognized and cleaved by the Drosha/DGCR8 heterodimer into pre-miRNA. The pre-miRNA hairpin loop is transported into the cytoplasm by Exportin-5, where its terminal loop is further cleaved by RNA endonuclease III Dicer to generate the mature duplex miRNA. Either strand can be processed into the final mature single-stranded miRNA, with the strand originating from the 5 'end of the transcript known as $5 \mathrm{p}$, while the strand originating from the 3 'end is known as 3p. Association of the mature miRNA duplex with Argonaut proteins (Ago) forms the RNA-induced silencing complex (RISC), where the guide strand of $\sim 22 \mathrm{nt}$ remains in Ago while the passenger strand gets degraded $^{[28,29]}$. Due to the short sequence length of miRNAs, and the fact that their partial complementary binding to multiple regions of target messenger RNAs (mRNAs) is sufficient to guide the RISC to its target genes, miRNAs are able to regulate the expression of a myriad of genes that play critical roles in controlling cellular processes, including autophagy and cancer metastasis.

\section{AUTOPHAGY-REGULATING MIRNAS IN CANCER METASTASIS}

Owing to the complexity and dynamic nature of autophagy, past literature that implicated a role of miRNAs in the regulation of autophagy have been highly discordant even in the same cell type. To reconcile and put things into perspective, we examined and discuss the opposing roles of miRNAregulated autophagy during the invasion phase versus the distant organ/tissue colonization phase of cancer metastasis.

At the onset of metastasis, cancer cells in the primary tumor first undergo EMT to enable the attached cancer cells to gain motile traits for invasion of the lymphovascular system. The role of autophagy in cancer cell motility and invasion was comprehensively reviewed by Mowers et al ${ }^{[23]}$. Suffice to say that many reports have now demonstrated the elevation in autophagy in cancer cells drives tumor dissemination, and this may in part be regulated by miRNAs. miRNAs that have been shown to regulate autophagy and cell migration/invasion in the context of cancer have been summarized in Table 1.

Upregulation in oncogenic miRNAs (oncomiRs) is known to promote tumorigenesis ${ }^{[29]}$ via modulation of gene expression of a variety of growth and survival pathways in cancer cells. For instance, elevated levels of miR-100 and miR-146 are found in cancer cells. Notably, these oncomiRs have been shown to be critical for the activation of pro-survival autophagy via suppression of the expression of autophagy inhibitory effectors like the mammalian target of rapamycin (mTOR) and Bcl2, respectively [Figure 1] $]^{[30,32,33]}$. miR-100 and miR-146 have also been shown to be key EMT effectors that promote metastasis ${ }^{[31,34]}$, with miR-100 proposed to act through HOXA inhibition ${ }^{[83]}$, and miR-146 via Notch2 inhibition ${ }^{[84]}$. Overexpression of 
Table 1. MiRNAs and their effects on autophagy and metastasis

\begin{tabular}{|c|c|c|c|c|c|}
\hline \multicolumn{6}{|c|}{ Effect in Autophagy } \\
\hline miRNA & Action & Gene target & Mechanism of action & Expression status in cancer & Effect in metastasis \\
\hline $\operatorname{miR}-100$ & Activator $^{[30]}$ & MTOR & Activates ULK1 initiation & Upregulated $^{[30]}$ & Activator $^{[30,31]}$ \\
\hline $\operatorname{miR}-146 a$ & Activator $^{[32]}$ & $B C L 2$ & Activates PI3K III nucleation & Upregulated $^{[33]}$ & Activator $^{[33,34]}$ \\
\hline $\operatorname{miR}-23 b$ & Inhibitor ${ }^{[35]}$ & $\begin{array}{l}\text { HMGB2 } \\
\text { ATG12 }\end{array}$ & $\begin{array}{l}\text { Inhibits PI3K III nucleation } \\
\text { Inhibits ATG12 conjugation }\end{array}$ & Downregulated ${ }^{[35,36]}$ & Inhibitor ${ }^{[36]}$ \\
\hline $\operatorname{miR}-24$ & Inhibitor $^{[37]}$ & $\begin{array}{l}\text { BCL2 } \\
\text { ATG4 }\end{array}$ & $\begin{array}{l}\text { Inhibits PI3K III nucleation } \\
\text { Inhibits LC3 conjugation }\end{array}$ & Downregulated $^{[38]}$ & Inhibitor ${ }^{[38]}$ \\
\hline $\operatorname{miR}-26 a / b$ & Inhibitor ${ }^{[39]}$ & ULK1 & Inhibits ULK1 initiation & Downregulated $^{[39,40]}$ & Inhibitor ${ }^{[40]}$ \\
\hline $\operatorname{miR}-30 a$ & Inhibitor ${ }^{[41]}$ & Beclin1 & Inhibits PI3K III nucleation & Downregulated $^{[42]}$ & Inhibitor ${ }^{[42]}$ \\
\hline $\operatorname{miR}-34$ & Inhibitor ${ }^{[43,44]}$ & $\begin{array}{l}\text { HMGB1 } \\
\text { ATG9a }\end{array}$ & $\begin{array}{l}\text { Inhibits PI3K III nucleation } \\
\text { Inhibits ATG9 cycling }\end{array}$ & Downregulated $^{[45]}$ & Inhibitor ${ }^{[46]}$ \\
\hline $\operatorname{miR}-125 a$ & Inhibitor ${ }^{[47]}$ & UVRAG & Inhibits PI3K III nucleation & Downregulated $^{[48]}$ & Inhibitor ${ }^{[48]}$ \\
\hline $\operatorname{miR}-141$ & Inhibitor ${ }^{[49]}$ & SIRT1 & $\begin{array}{l}\text { Inhibits ATG12 and LC3 } \\
\text { conjugation }\end{array}$ & Downregulated $^{[50]}$ & Inhibitor ${ }^{[50,51]}$ \\
\hline $\operatorname{miR}-143$ & Inhibitor $^{[52]}$ & $A T G 2 B$ & Inhibits ATG9 cycling & Downregulated $^{[53]}$ & Inhibitor ${ }^{[53]}$ \\
\hline $\operatorname{miR}-199$ & Inhibitor ${ }^{[54,55]}$ & $\begin{array}{l}\text { ATG14 } \\
\text { Rab7A }\end{array}$ & $\begin{array}{l}\text { Inhibits PI3K III nucleation } \\
\text { Inhibits autophagosome and } \\
\text { lysosome fusion }\end{array}$ & Downregulated $^{[56]}$ & Inhibitor ${ }^{[56]}$ \\
\hline $\operatorname{miR}-200$ & Inhibitor ${ }^{[57]}$ & ATG12 & Inhibits ATG12 conjugation & Downregulated $^{[58]}$ & Inhibitor ${ }^{[58,59]}$ \\
\hline $\operatorname{miR}-520$ & Inhibitor ${ }^{[60]}$ & ATG7 & $\begin{array}{l}\text { Inhibits ATG12 and LC3 } \\
\text { conjugation }\end{array}$ & Downregulated $^{[60]}$ & Inhibitor ${ }^{[61]}$ \\
\hline $\operatorname{miR}-21$ & Inhibitor ${ }^{[62,63]}$ & $\begin{array}{l}\text { Rab11A } \\
\text { PTEN }\end{array}$ & $\begin{array}{l}\text { Inhibits ULK1 initiation and ATG9 } \\
\text { cycling }\end{array}$ & Upregulated $^{[64]}$ & Activator $^{[64]}$ \\
\hline $\operatorname{miR}-29 a$ & Inhibitor ${ }^{[65]}$ & ATG9A & Inhibits ATG9 and its cycling & Upregulated $^{[66]}$ & Activator ${ }^{[66]}$ \\
\hline $\operatorname{miR}-93$ & Inhibitor ${ }^{[67,68]}$ & $\begin{array}{l}\text { PTEN } \\
\text { ATG16L1 }\end{array}$ & $\begin{array}{l}\text { Inhibits ULK1 initiation } \\
\text { Inhibits ATG12 conjugation }\end{array}$ & Upregulated $^{[67]}$ & Activator $^{[67,69]}$ \\
\hline $\operatorname{miR}-214$ & Inhibitor $^{[70]}$ & $\begin{array}{l}\text { PTEN } \\
\text { ATG12 }\end{array}$ & $\begin{array}{l}\text { Inhibits ULK1 initiation } \\
\text { Inhibits ATG12 conjugation }\end{array}$ & Upregulated $^{[71]}$ & Activator $^{[71]}$ \\
\hline $\operatorname{miR}-221 / 222$ & Inhibitor ${ }^{[72]}$ & ATG12 & Inhibits ATG12 conjugation & Upregulated $^{[72,73]}$ & Activator $^{[73]}$ \\
\hline let-7 & Activator $^{[74]}$ & MTOR & Activate ULK1 initiation & Downregulated $^{[75]}$ & Inhibitor $^{[75]}$ \\
\hline $\operatorname{miR}-7$ & Activator $^{[76]}$ & MTOR & Activates ULK1 initiation & Downregulated $^{[77]}$ & Inhibitor ${ }^{[77]}$ \\
\hline $\operatorname{miR}-16$ & Activator $^{[78]}$ & MTOR & Activates ULK1 initiation & Downregulated $^{[79]}$ & Inhibitor ${ }^{[79]}$ \\
\hline $\operatorname{miR}-205$ & Activator $^{[80,81]}$ & PTEN & Activates ULK1 intiation & Downregulated $^{[82]}$ & Inhibitor ${ }^{[82]}$ \\
\hline
\end{tabular}

these oncomiRs provides several advantages to the invading cancer cells. Firstly, an increase in autophagy flux helps DTCs to resist anoikis following detachment from the primary tumor as well as to enable DTCs to gain access to nutrients to survive the arduous journey of traversing to distant organs/tissues. In addition, they concurrently change gene expression patterns in the cancer cells to allow these cells to become mesenchymal and thus more motile to invade distant sites.

Apart from oncomiRs, the loss of tumor-suppressive miRNAs has also been shown to drive cell invasion and migration [Figure 1]. Several autophagy-inhibiting miRNAs, namely, miR-23b, miR-24, miR-26, miR-30a, miR-34, miR-125a, miR-141, miR-143, miR-199, miR-200 and miR-520 are found to be downregulated in cancer. Notably, these miRNAs are also known MET effectors ${ }^{[35-43,45-61,85,86]}$. As opposed to the primary role(s) of oncomiRs, we speculate that the loss of these miRNAs allows for higher autophagy flux and suppression of MET in the DTCs to help them adapt to unfavorable settlement sites.

Intriguingly, there exists a group of miRNAs that appear to block autophagy and yet promote EMT. These include oncogenic miR-21, miR-29a, miR-93, miR-214 and the miR-221/222 cluster that are highly expressed in cancer [Figure 2] $]^{[62-73,87]}$. It seems counter-intuitive that successful distant organ/tissue invasion can be achieved for cancer cells without autophagy, but it remains plausible that the effects of these miRNAs might be context-dependent. For example, overexpression of miR-21 and miR-93 have been shown to induce metastasis by targeting PTEN ${ }^{[64,69]}$, which in turn may promote the blockade of autophagy via activating the PI3K-Akt-mTOR signaling cascade. Since one miRNA can target many different genes, the biological 


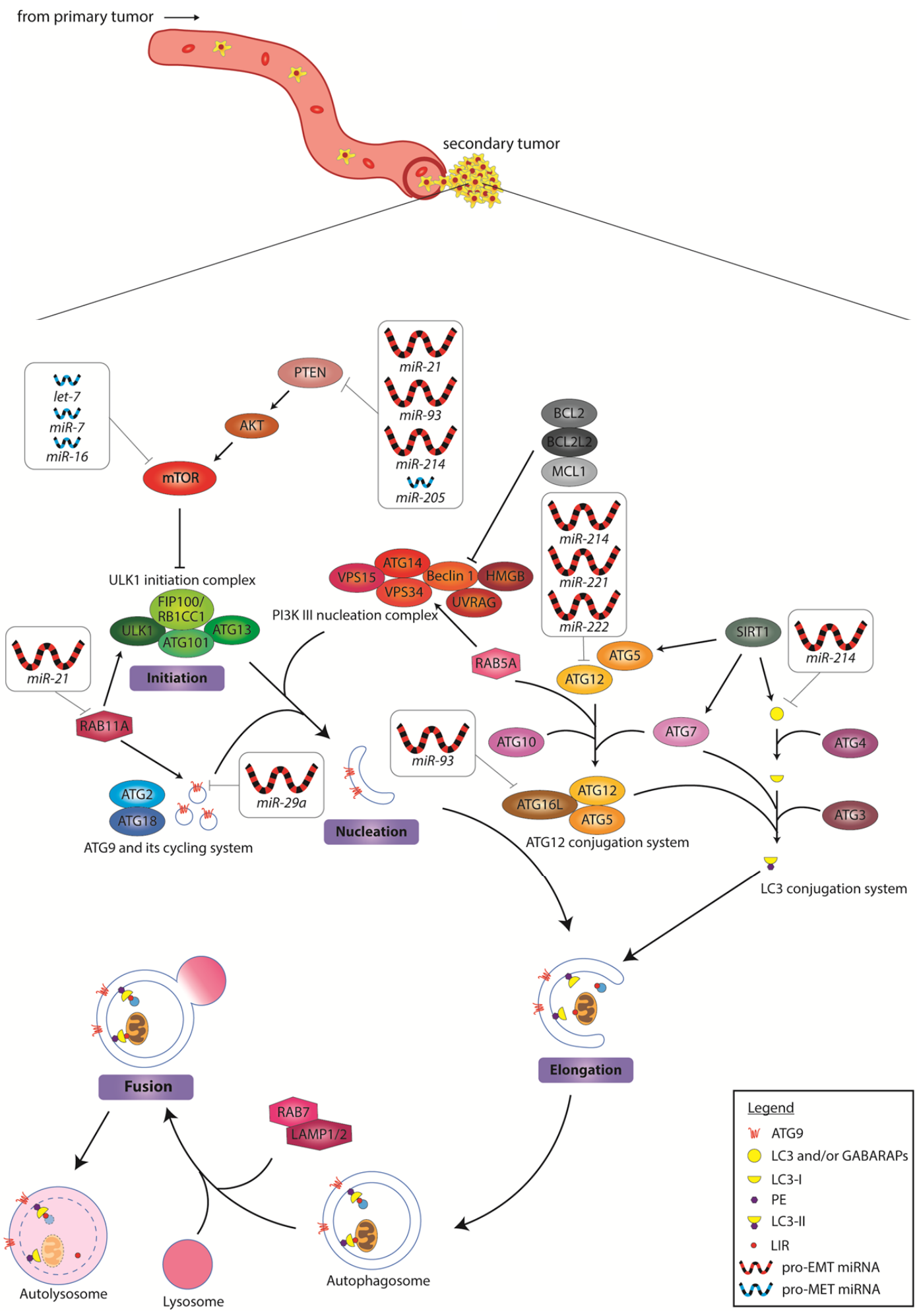

Figure 2. MiRNA inhibition of autophagy during secondary tumor formation. In the colonization process, autophagy is inhibited either by upregulation of anti-autophagy miRNAs, or the downregulation of the pro-autophagy miRNAs. This process promotes the reestablishment of an epithelial cell state for developing into macrometastasis. Upregulated pro-EMT miRNAs are depicted as large red icons, while downregulated pro-MET miRNAs are depicted as small blue icons. EMT: epithelial-to-mesenchymal transition; MET: mesenchymal-to-epithelial transition 
context should also be taken into consideration in reconciling the opposing EMT-promoting and antiautophagy effects of miRNA-dependent loss of PTEN. Could miR-21 and miR-93 also regulate other targets concurrently to yield the phenotype observed? We also noted that several of these studies demonstrate induction of cell migration/invasion by overexpressing miR-21 or miR-93 in cancer cells grown in the tissue culture setting ${ }^{[64,67]}$. Unlike the circulating DTCs in a living organism, cancer cells cultured in vitro are never short of nutrient supply. It is perhaps not surprising that the need to trigger pro-survival autophagy in these cancer cells might be over-written by external culture conditions in vitro. Alternatively, miRNAs like miR-21 and miR-93 may serve as sensors of favorable distant niches. As the DTCs extravasate at their destination sites, these miRNAs could respond to the new environment that provides nutrients to fuel the transition from micro- to macro-metastasis. Similarly, there exists tumor suppressive miRNAs that have been implicated in autophagy activation but yet inhibit cancer growth and metastasis. These miRNAs, including let-7, miR-7, miR-16 and miR-205, have been shown to be less abundant in tumors ${ }^{[74-82]}$. Their absence in DTCs at micrometastasis sites might be crucial to cell survival, as autophagy genes will remain activated to ensure nutrient supply until conditions of the new environment turn favorable for macrometastasis.

\section{PRECISION TARGETING OF MIRNA-AUTOPHAGY SIGNALING NODES BY SMALL MOLECULE AND RNA-BASED THERAPEUTICS}

To date, cytotoxic chemotherapy remains the most widely used neoadjuvant or adjuvant treatment modality against cancers despite its association with a host of unpredictable side effects ${ }^{[88]}$. In addition, a recent study demonstrates that cytotoxic chemotherapeutics broadly used in neoadjuvant cancer therapy elicit tumour-derived extracellular vesicles with enhanced pro-metastatic capacity ${ }^{[89]}$. Hence, precise targeting of metastasis-enabling cellular processes, such as autophagy, is warranted and could synergize with chemotherapy to maximize therapeutic efficacy. For instance, small molecule inhibitor targeting of focal adhesion kinase (commonly known as FAK) activates the SRC kinase and thereby inhibits autophagy, which in turn, prevents the migration of SRC-driven metastatic tumor cells ${ }^{[90,91]}$. Given the complexity of signaling pathways driving metastasis, a systematic framework for the development of effective antimetastatic drugs has recently been proposed ${ }^{[92]}$.

Of late, RNA-based therapies (antisense, siRNAs, aptamers, miRNA mimics/anti-miRs and synthetic mRNA) have shown great promise as powerful adjuncts to the drug developer's existing toolbox of small molecules and biologics ${ }^{[93]}$. Notably, miRNA-based therapeutics gained much attention in translational medicine and are currently tested in various Phase I and II clinical trials. miRNA-based therapeutics is broadly categorized as miRNA mimics and inhibitors of miRNAs (also known as anti-miRs or antagomiRs). miRNA mimics, in the form of synthetic double-stranded small RNA molecules that match the corresponding miRNA sequence, are aimed at replenishing the lost miRNA expression in diseases. Most of the commercial miRNA mimics are often modified by methylation of the passenger strand to increase their stability. AntagomiRs, on the other hand, are antisense oligonucleotides that are singlestranded and can be chemically-modified (with locked nucleic acids or with 2'-O-methoxyethylribonucle oside) to increase affinity for their miRNA targets to block their functions ${ }^{[94]}$. To prevent degradation by RNases in biofluids or in the endocytic compartment of cells, these miRNA therapeutics are frequently stabilized by oligonucleotide chemistry (as mentioned above or by adding phosphorothioate-like groups) or encapsulated in delivery vehicles, such as poly(lactide-co-glycolide) particles, neutral lipid emulsions, synthetic polyethylenimine, cyclodextrin, chitosan, etc., ${ }^{[95]}$. For instance, epidermal growth factor (EGFR)targeted, EnGeneIC delivery vehicle-packaged miR-16 mimics have been tested in a Phase I clinical trial (NCT02369198) in which acceptable safety profile and on-target activity of these miR-16 mimics have been demonstrated in patients with malignant pleural mesothelioma ${ }^{[96]}$. In addition, two other clinical trials (NCT02862145 and NCT01829971), involving the use of miR-34a mimics to treat primary liver cancer, small 
cell lung cancer (SCLC), lymphoma, multiple myeloma, renal cell carcinoma and melanoma, also showed promising anti-cancer results ${ }^{[97]}$. Given that a subset of miRNAs mediate the crosstalk between autophagy and metastatic states of cancer cells, we postulate that the miRNA-autophagy-metastasis axis of cancer could be effectively targeted by the precise in vivo delivery of specific combinations of miRNA therapeutics (involving miRNAs depicted in Figures 1 and 2), which could improve the clinical trajectory of late stage cancers. Alternatively, blockade of the biogenesis of oncomiRs via small-molecule RNA ligands has also been reported to be a feasible anti-cancer approach ${ }^{[98]}$. These small molecules consist of high affinity RNA binding motives, including the aminoglycoside neomycin and different natural and artificial nucleobases, that target pri-miRNAs of oncogenic miR-372/miR-373 and inhibit Dicer-mediated maturation of these miRNAs to elicit anti-proliferative effects on gastric cancer cells.

Apart from miRNA therapeutics, siRNA therapeutics that target a number of driver oncogenes like $c-M y c$, PD-L1/2, mutant KRAS, PKN3, EphA2, LMP2/7, MECL1, APN401 and PLK1 have also been shown to exhibit on-target anti-cancer effects and elicit little or no overt toxicities in humans in a number of Phase I clinical trials (NCT00306904, NCT02314052, NCT02528682, NCT01676259, NCT01808638, NCT01591356, NCT00672542, NCT02166255 and NCT01437007). We envisage that delivery of siRNAs targeting genes that are important for autophagy and metastasis of cancer cells could also be a viable therapeutic route to combat late stage cancers.

\section{CONCLUSION}

Alterations in key cellular processes, including cell adhesive properties and autophagy, promote tumor cell dissemination and colonization at distant organs/tissues. These processes are mediated by a vast network of miRNAs that has the propensity to crosstalk with each other. Striking this Achilles heel of cancer precisely via specific combinations of miRNA therapeutics could dramatically improve the survival outcomes of cancer patients. Future work should be focused on tissue-specific targeting delivery vehicles, which carry miRNA therapeutics or other payloads, so as to avoid potential toxicities and off-target effects.

\section{DECLARATIONS}

\section{Authors' contributions}

Manuscript writing: Eng GWL, Kok VJT, Cheong JK

\section{Availability of data and materials}

Not applicable.

\section{Financial support and sponsorship}

Eng GWL is supported by the Singapore Ministry of Education Postdoctoral Fellowship. Kok VJT is supported by the Singapore Ministry of Education (MOE) Academic Research Fund (AcRF) Tier 2 grant (MOE2016-T2-2-052). Cheong JK is supported by the Singapore MOE AcRF Tier 2 grant (MOE2016-T2-2-052) and a startup grant from the Medical Sciences Cluster of Yong Loo Lin School of Medicine, National University of Singapore.

\section{Conflicts of interest}

All authors declared that there are no conflicts of interest.

\section{Ethical approval and consent to participate}

Not applicable. 


\section{Consent for publication}

Not applicable.

\section{Copyright}

(c) The Author(s) 2019.

\section{REFERENCES}

1. Hawkes N. Cancer survival data emphasise importance of early diagnosis. BMJ 2019;364:1408.

2. Hosseini H, Obradovic MMS, Hoffmann M, Harper KL, Sosa MS, et al. Early dissemination seeds metastasis in breast cancer. Nature 2016;540:552-8.

3. Wan L, Pantel K, Kang Y. Tumor metastasis: moving new biological insights into the clinic. Nat Med 2013;19:1450-64.

4. Peng JM, Bera R, Chiou CY, Yu MC, Chen TC, et al. Actin cytoskeleton remodeling drives epithelial-mesenchymal transition for hepatoma invasion and metastasis in mice. Hepatology 2018;67:2226-43.

5. Quail DF, Joyce JA. Microenvironmental regulation of tumor progression and metastasis. Nat Med 2013;19:1423-37.

6. Thiery JP, Acloque H, Huang RY, Nieto MA. Epithelial-mesenchymal transitions in development and disease. Cell 2009;139:871-90.

7. Massague J, Obenauf AC. Metastatic colonization by circulating tumour cells. Nature 2016;529:298-306.

8. Fischer KR, Durrans A, Lee S, Sheng J, Li F, et al. Epithelial-to-mesenchymal transition is not required for lung metastasis but contributes to chemoresistance. Nature 2015;527:472-6.

9. Cazet AS, Hui MN, Elsworth BL, Wu SZ, Roden D, et al. Targeting stromal remodeling and cancer stem cell plasticity overcomes chemoresistance in triple negative breast cancer. Nat Commun 2018;9:2897.

10. Yin Z, Pascual C, Klionsky D. Autophagy: machinery and regulation.Microb Cell 2016;3:588-96.

11. Kim J, Kundu M, Viollet B, Guan KL. AMPK and mTOR regulate autophagy through direct phosphorylation of Ulk1. Nat Cell Biol 2011;13:132-41.

12. Parzych KR, Klionsky DJ. An Overview of Autophagy: Morphology, Mechanism, and Regulation. Antioxid Redox Signal 2014;20:460-73.

13. Feng Y, He D, Yao Z, Klionsky DJ. The machinery of macroautophagy 24 The machinery of macroautophagy. Cell Res 2014;24:24-41.

14. Lee YK, Lee JA. Role of the mammalian ATG8/LC3 family in autophagy: Differential and compensatory roles in the spatiotemporal regulation of autophagy. BMB Rep 2016;49:424-30.

15. Nazio F, Bordi M, Cianfanelli V, Locatelli F, Cecconi F. Autophagy and cancer stem cells: molecular mechanisms and therapeutic applications. Cell Death Differ 2019;26:690-702.

16. Gugnoni M, Sancisi V, Manzotti G, Gandolfi G, Ciarrocchi A. Autophagy and epithelial-mesenchymal transition: an intricate interplay in cancer. Cell Death Dis 2016;7:e2520.

17. Galavotti S, Bartesaghi S, Faccenda D, Shaked-Rabi M, Sanzone S, et al. The autophagy-associated factors DRAM1 and p62 regulate cell migration and invasion in glioblastoma stem cells. Oncogene 2013;32:699-712.

18. Frisch SM, Francis H. Disruption of epithelial cell-matrix interactions induces apoptosis. J Cell Biol 1994;124:619-26.

19. Meredith JE, Fazeli B, Schwartz MA, Schwartz MA. The extracellular matrix as a cell survival factor. Mol Biol Cell 1993;4:953-61.

20. Fung C, Lock R, Gao S, Salas E, Debnath J. Induction of Autophagy during Extracellular Matrix Detachment Promotes Cell Survival. Mol Biol Cell 2008;19:797-806.

21. Kenific CM, Thorburn A, Debnath J. Autophagy and metastasis: Another double-edged sword. Curr Opin Cell Biol 2010;22:241-5.

22. Peng YF, Shi YH, Ding ZB, Ke AW, Gu CY, et al. Autophagy inhibition suppresses pulmonary metastasis of HCC in mice via impairing anoikis resistance and colonization of HCC cells. Autophagy 2013;9:2056-68.

23. Mowers EE, Sharifi MN, Macleod KF. Autophagy in cancer metastasis. Oncogene 2017;36:1619-30.

24. Chen HT, Liu H, Mao MJ, Tan Y, Mo XQ, et al. Crosstalk between autophagy and epithelial-mesenchymal transition and its application in cancer therapy. Mol Cancer 2019;18:101.

25. Banyard J, Bielenberg DR. The role of EMT and MET in cancer dissemination. Connect Tissue Res 2015;56:403-13.

26. Barnard RA, Regan DP, Hansen RJ, Maycotte P, Thorburn A, et al. Autophagy Inhibition Delays Early but Not Late-Stage Metastatic Disease. J Pharmacol Exp Ther 2016;358:282-93.

27. Paul P, Chakraborty A, Sarkar D, Langthasa M, Rahman M, et al. Interplay between miRNAs and human diseases. J Cell Physiol 2018;233:2007-18

28. O'Brien J, Hayder H, Zayed Y, Peng C. Overview of microRNA biogenesis, mechanisms of actions, and circulation. Front Endocrinol (Lausanne) 2018;9:402.

29. Di Leva G, Garofalo M, Croce CM. MicroRNAs in Cancer. Annu Rev Pathol 2014;9:287-314.

30. Wang W, Liu Y, Guo J, He H, Mi X, et al. miR-100 maintains phenotype of tumor-associated macrophages by targeting mTOR to promote tumor metastasis via Stat5a/IL-1ra pathway in mouse breast cancer. Oncogenesis 2018;7:97.

31. Ottaviani S, Stebbing J, Frampton AE, Zagorac S, Krell J, et al. TGF- $\beta$ induces miR-100 and miR-125b but blocks let-7a through LIN28B controlling PDAC progression. Nature Communications 2018;9:10.

32. Zhang F, Wang J, Chu J, Yang C, Xiao H, et al. MicroRNA-146a Induced by Hypoxia Promotes Chondrocyte Autophagy through Bcl2. Cell Physiol Biochem 2015;37:1442-53. 
33. Yang L, Zhao G, Wang F, Li C, Wang X. Hypoxia-Regulated miR-146a Targets Cell Adhesion Molecule 2 to Promote Proliferation, Migration, and Invasion of Clear Cell Renal Cell Carcinoma. Cell Physiol Biochem 2018;49:920-931.

34. Zhou W, Thiery JP. Loss of Git2 induces epithelial-mesenchymal transition by miR146a-Cnot6L-controlled expression of Zeb1. J Cell Sci 2013;126:2740-6.

35. An Y, Zhang Z, Shang Y, Jiang X, Dong J, et al. miR-23b-3p regulates the chemoresistance of gastric cancer cells by targeting ATG12 and HMGB2. Cell Death Dis 2015;6:e1766.

36. Cao J, Liu J, Long J, Fu J, Huang L, et al. microRNA-23b suppresses epithelial-mesenchymal transition (EMT) and metastasis in hepatocellular carcinoma via targeting Pyk2. Biomed Pharmacother 2017;89:642-50.

37. Pan B, Chen Y, Song H, Xu Y, Wang R, et al. Mir-24-3p downregulation contributes to VP16-DDP resistance in small-cell lung cancer by targeting ATG4A. Oncotarget 2015;6:317-31.

38. Kang H, Rho JG, Kim C, Tak H, Lee H, et al. The MIR-24-3p/p130Cas: A novel axis regulating the migration and invasion of cancer cells. Sci Rep 2017;7:44847.

39. Jin F, Wang Y, Li M, Zhu Y, Liang H, et al. MiR-26 enhances chemosensitivity and promotes apoptosis of hepatocellular carcinoma cells through inhibiting autophagy.Cell Death Dis 2018;8:e2540.

40. Chang L, Li K, Guo T. miR-26a-5p suppresses tumor metastasis by regulating EMT and is associated with prognosis in HCC. Clin Transl Oncol 2017;19:695-703.

41. Yang X, Bai F, Xu Y, Chen Y, Chen L. Intensified Beclin-1 Mediated by Low Expression of Mir-30a-5p Promotes Chemoresistance in Human Small Cell Lung Cancer. Cell Physiol Biochem 2017;43;1126-39.

42. Wei W, Yang Y, Cai J, Cui K, Li RX, et al. MiR-30a-5p Suppresses Tumor Metastasis of Human Colorectal Cancer by Targeting ITGB3. Cell Physiol Biochem 2016;39;1165-76.

43. Liu L, Ren W, Chen K. MiR-34a promotes apoptosis and inhibits autophagy by targeting HMGB1 in acute myeloid leukemia cells. Cell Physiol Biochem 2017;41;1981-92.

44. Pang J, Xiong H, Lin P, Lai L,Yang H, et al. Activation of miR-34a impairs autophagic flux and promotes cochlear cell death via repressing ATG9A: implications for age-related hearing loss. Cell Death Dis 2017;8:e3079.

45. Zhang X, Ai F, Li X, Tian L, Wang X, et al. MicroRNA-34a suppresses colorectal cancer metastasis by regulating notch signaling. Oncol Lett 2017;14:2325-33.

46. Siemens H, Jackstadt R, Hünten S, Kaller M, Menssen A, et al. Cell Cycle miR-34 and SNAIL form a double-negative feedback loop to regulate epithelial-mesenchymal transitions. Cell Cycle 2011;10:4256-71.

47. Kim JK, Yuk JM, Kim SY, Kim TS, Jin HS, et al. MicroRNA-125a Inhibits Autophagy Activation and Antimicrobial Responses during Mycobacterial Infection. J Immunol 2015;194:355-65.

48. Fan Z, Cui H, Xu X, Lin Z, Zhang X, et al. MiR-125a suppresses tumor growth, invasion and metastasis in cervical cancer by targeting STAT3. Oncotarget 2015;6:25266-80.

49. Yang Y, Liu Y, Xue J, Yang Z, Shi Y, et al. MicroRNA-141 Targets Sirt1 and Inhibits Autophagy to Reduce HBV Replication. Cell Physiol Biochem 2017;41:310-22.

50. Huang S, Wa Q, Pan J, Peng X, Ren D, et al. Downregulation of miR-141-3p promotes bone metastasis via activating NF- $\kappa \mathrm{B}$ signaling in prostate cancer. J Exp Clin Cancer Res 2017;36:173.

51. Liu C, Liu R, Zhang D, Deng Q, Liu B, et al. MicroRNA-141 suppresses prostate cancer stem cells and metastasis by targeting a cohort of pro-metastasis genes. Nat Commun 2017;8:14270.

52. Wei J, Ma Z, Li Y, Zhao B, Wang D, et al. MiR-143 inhibits cell proliferation by targeting autophagy-related 2B in non-small cell lung cancer H1299 cells. Mol Med Rep 2015;11:571-6.

53. Lei C, Du F, Sun L, Li T, Li T, et al. MIR-143 \& MIR-145 inhibit gastric cancer cell migration \& metastasis by suppressing MYO6. Cell Death Dis 2017;8:e3101.

54. Li B, Wu X, Chen H, Zhuang C, Zhang Z, et al. MiR199a-5p inhibits hepatic insulin sensitivity via suppression of ATG14-mediated autophagy article. Cell Death Dis 2018;9:405.

55. Aranda JF, Rathjen S, Johannes L, Fernández-Hernando C, Francisco Aranda J. MiR-199a-5p attenuates retrograde transport and protects against toxin-2 induced inhibition of protein biosynthesis. Mol Cell Biol 2018;38:e00548-17.

56. Zhan Y, Zheng N, Teng F, Bao L, Liu F, et al. MiR-199a/b-5p inhibits hepatocellular carcinoma progression by post-transcriptionally suppressing ROCK1. Oncotarget 2017;8:67169-80.

57. Pan B, Feng B, Chen Y, Huang G, Wang R, et al. MiR-200b regulates autophagy associated with chemoresistance in human lung adenocarcinoma. Oncotarget LLC 2015;6:32805-20.

58. Park SM, Gaur AB, Lengyel E, Peter ME. The miR-200 family determines the epithelial phenotype of cancer cells by targeting the E-cadherin repressors ZEB1 and ZEB2. Genes Dev 2008;22:894-907.

59. Lai HH, Li JN, Wang MY, Huang HY, Croce CM, et al. HIF-1 $\alpha$ promotes autophagic proteolysis of dicer and enhances tumor metastasis. J Clin Invest 2018;128:625-43.

60. Gao AM, Zhang XY, Hu JN, Ke ZP. Apigenin sensitizes hepatocellular carcinoma cells to doxorubic through regulating miR-520b/ ATG7 axis. Chem Biol Interact 2018;280:45-50.

61. Lu YC, Cheng AJ, Lee LY, You GR, Li YL, et al. MiR-520b as a novel molecular target for suppressing stemness phenotype of headneck cancer by inhibiting CD44. Sci Rep 2017;7:2042.

62. Liu X, Hong Q, Wang Z, Yu Y, Zou X, et al. MiR-21 inhibits autophagy by targeting Rab11a in renal ischemia/reperfusion. Experimental Cell Res 2015;338:64-9. 
63. Wang WJ, Yang W, Ouyang ZH, Xue JB, Li XL, et al. MiR-21 promotes ECM degradation through inhibiting autophagy via the PTEN/akt/mTOR signaling pathway in human degenerated NP cells. Biomed Pharmacother 2018;99:725-34.

64. Liu ZL, Wang H, Liu J, Wang ZX. MicroRNA-21 (miR-21) expression promotes growth, metastasis, and chemo- or radioresistance in non-small cell lung cancer cells by targeting PTEN. Mol Cell Biochem 2013;372:35-45.

65. Xu Y, Yang J, Li F, Lian G, Ouyang M. MiR-29a inhibited intestinal epithelial cells autophagy partly by decreasing ATG9A in ulcerative colitis. Anti-Cancer Drug 2018;29:652-9.

66. Tang W, Zhu Y, Gao J, Fu J, Liu C, et al. MicroRNA-29a promotes colorectal cancer metastasis by regulating matrix metalloproteinase 2 and E-cadherin via KLF4. Br J Cancer 2013;110:450-8.

67. Li N, Miao Y, Shan Y, Liu B, Li Y, et al. MiR-106b and miR-93 regulate cell progression by suppression of PTEN via PI3K/Akt pathway in breast cancer. Cell Death Dis 2017;8:e2796.

68. Lu C, Chen J, Xu HG, Zhou X, He Q, et al. MIR106B and MIR93 prevent removal of bacteria from epithelial cells by disrupting ATG16L1-mediated autophagy. Gastroenterology 2014;146:188-99.

69. Chu S, Liu G, Xia P, Chen G, Shi F, et al. miR-93 and PTEN: Key regulators of doxorubicin-resistance and EMT in breast cancer. Oncol Rep 2017;38:2401-7.

70. Hu JL, He GY, Lan XL, Zeng ZC, Guan J, et al. Inhibition of ATG12-mediated autophagy by miR-214 enhances radiosensitivity in colorectal cancer. Oncogenesis 2018;7:16.

71. Liu J, Li D, Dang L, Liang C, Guo B, et al. Osteoclastic miR-214 targets TRAF3 to contribute to osteolytic bone metastasis of breast cancer. Sci Rep 2017;7:40487.

72. Xu J, Su Y, Xu A, Fan F, Mu S, et al. miR-221/222-Mediated Inhibition of Autophagy Promotes Dexamethasone Resistance in Multiple Myeloma. Mol Ther 2019;27:559-70.

73. Liang YK, Lin HY, Dou XW, Chen M, Wei XL, et al. MiR-221/222 promote epithelial-mesenchymal transition by targeting Notch3 in breast cancer cell lines. NPJ Breast Cancer 2018;4:20.

74. Dubinsky AN, Ghosh Dastidar S, Hsu CL, Zahra R, Djakovic S, et al. Let-7 coordinately suppresses components of the amino acid sensing pathway to repress mTORC1 and induce autophagy. Cell Metab 2014;20:626-38 .

75. Ling R, Zhou Y, Zhou L, Dai D, Wu D, et al. Lin28/microRNA-let-7a promotes metastasis under circumstances of hyperactive Wnt signaling in esophageal squamous cell carcinoma. Mol Med Rep 2018;17:5265-71.

76. Fang Y, Xue JL, Shen Q, Chen J, Tian L. MicroRNA-7 inhibits tumor growth and metastasis by targeting the phosphoinositide 3-kinase/Akt pathway in hepatocellular carcinoma. Hepatology 2012;55:1852-62.

77. Kong X, Li G, Yuan Y, He Y, Wu X. MicroRNA-7 Inhibits Epithelial-to-Mesenchymal Transition and Metastasis of Breast Cancer Cells via Targeting FAK Expression. PLoS One 2012;7:e41523.

78. Janaki Ramaiah M, Lavanya A, Honarpisheh M, Zarea M, Bhadra U, et al. MiR-15/16 complex targets p70S6 kinase1 and controls cell proliferation in MDA-MB-231 breast cancer cells. Gene 2014;552:255-64.

79. Zhang H, Yang K, Ren T, Huang Y, Tang X, et al. MiR-16-5p inhibits chordoma cell proliferation, invasion and metastasis by targeting Smad3 article. Cell Death Dis 2018;9:680.

80. Pennati M, Lopergolo A, Profumo V, De Cesare M, Sbarra S, et al. miR-205 impairs the autophagic flux and enhances cisplatin cytotoxicity in castration-resistant prostate cancer cells. Biochem Pharmacol 2014;87:579-97.

81. Zhuo Z, Yu H, Zhuo Z,Yu H, Zhuo Z, et al. miR-205 inhibits cell growth by targeting AKT-mTOR signaling in progesterone-resistant endometrial cancer Ishikawa cells. Oncotarget 2017;8:28042-51.

82. Gulei D, Magdo L, Jurj A, Raduly L, Cojocneanu-Petric R, et al. The silent healer: miR-205-5p up-regulation inhibits epithelial to mesenchymal transition in colon cancer cells by indirectly up-regulating E-cadherin expression. Cell Death Dis 2018;9:66.

83. Chen D, Sun Y, Yuan Y, Han Z, Zhang P, et al. miR-100 Induces Epithelial-Mesenchymal Transition but Suppresses Tumorigenesis, Migration and Invasion. PLoS Genet 2014;10:e1004177.

84. Wang C, Zhang W, Zhang L, Chen X, Liu F, et al. miR-146a-5p mediates epithelial-mesenchymal transition of oesophageal squamous cell carcinoma via targeting Notch2. Brit J Cancer 2016;115:1548-54.

85. Zhang MX, Zhang J, Zhang H, Tang H. MiR-24-3p suppresses malignant behavior of lacrimal adenoid cystic carcinoma by targeting PRKCH to regulate p53/p21 pathway. PLoS ONE 2016;11:e0158433.

86. Chao CC, Wu PH, Huang HC, Chung HY, Chou YC, et al. Downregulation of miR-199a/b-5p is associated with GCNT2 induction upon epithelial-mesenchymal transition in colon cancer. FEBS Lett 2017;591:1902-17.

87. Bornachea O, Santos M, Martínez-Cruz AB, García-Escudero R, Dueñas M, et al. EMT and induction of miR-21 mediate metastasis development in Trp53-deficient tumours. Sci Rep 2012;2:434.

88. Vera-Ramirez L, Vodnala SK, Nini R, Hunter KW, Green JE. Autophagy promotes the survival of dormant breast cancer cells and metastatic tumour recurrence. Nat Commun 2018;9:1944.

89. DeVita VT Jr, Chu E. A history of cancer chemotherapy. Cancer Res 2008;68:8643-53.

90. Keklikoglou I, Cianciaruso C, Guc E, Squadrito ML, Spring LM, et al. Chemotherapy elicits pro-metastatic extracellular vesicles in breast cancer models. Nat Cell Biol 2019;21:190-202.

91. Schoenherr C, Byron A, Sandilands E, Paliashvili K, Baillie GS, et al. Ambral spatially regulates Src activity and Src/FAK-mediated cancer cell invasion via trafficking networks. Elife 2017;6:e23172.

92. Sharifi MN, Mowers EE, Drake LE, Collier C, Chen H, et al. Autophagy Promotes Focal Adhesion Disassembly and Cell Motility of Metastatic Tumor Cells through the Direct Interaction of Paxillin with LC3. Cell Rep 2016;15:1660-72.

93. Anderson RL, Balasas T, Callaghan J, Coombes RC, Evans J, et al. A framework for the development of effective anti-metastatic 
agents. Nat Rev Clin Oncol 2019;16:185-204.

94. Huggett B, Paisner K. The commercial tipping point. Nat Biotechnol 2017;35:708-9.

95. Rupaimoole R, Slack FJ. MicroRNA therapeutics: towards a new era for the management of cancer and other diseases. Nat Rev Drug Discov 2017;16:203-22.

96. van Zandwijk N, Pavlakis N, Kao SC, Linton A, Boyer MJ, et al. Safety and activity of microRNA-loaded minicells in patients with recurrent malignant pleural mesothelioma: a first-in-man, phase 1, open-label, dose-escalation study. Lancet Oncol 2017;18:1386-96.

97. Beg MS, Brenner AJ, Sachdev J, Borad M, Kang YK, et al. Phase I study of MRX34, a liposomal miR-34a mimic, administered twice weekly in patients with advanced solid tumors. Invest New Drugs 2017;35:180-8.

98. Vo DD, Staedel C, Zehnacker L, Benhida R, Darfeuille F, et al. Targeting the Production of Oncogenic MicroRNAs with Multimodal Synthetic Small Molecules. ACS Chem Biol 2014;9:711-21. 